UN S/2004/616, Report of the Secretary-General on the Rule of Law and Transitional Justice in Conflict and Post-Conflict Societies, 23 August 2004, available at http://www.un.org/ Docs/sc/sgrep04.html (last visited 30 June 2013).

Waddell, N. \& Clark, P. (eds.) 2008, Courting Conflict? Justice, Peace and the ICC in Africa, London: Royal African Society.
Wippman, D. 2004, The International Criminal Court, in: Reus-Smit, C. (ed) 2004, The Politics of International Law, Cambridge: Cambridge University Press, 151-188.

Zacklin, R., 2004, The Failings of Ad Hoc International Tribunals, In: Journal of International Criminal Justice 2, 541-542.

\title{
Völkermord abschaffen: Ein Gedankenexperiment
}

\author{
Mayeul Hiéramente*
}

\begin{abstract}
The 1948 Genocide Convention is rightly considered a milestone in the development of international criminal law. Due to an imperfect definition, overblown expectations by lawyers, politicians and the general public, and enormous difficulties in proving acts of genocide, the law of genocide might have turned into an obstacle and burden to the evolution of international law and the functioning of institutions like the International Criminal Court. It is argued that there is no need for the law of genocide in the $21^{\text {st }}$ century, and that the goals of international (criminal) law can better be pursued by focusing on the broader and more practical definition of crimes against humanity.
\end{abstract}

Keywords: International Criminal Court, genocide, crimes against humanity

Internationaler Strafgerichtshof, Völkermord, Verbrechen gegen die Menschlichkeit

\section{Ein Blick zurück}

$\mathrm{R}$ ückblicke auf das Wirken einer Institution wie den Internationalen Strafgerichtshof (IStGH) sind kein leichtes Unterfangen. Persönliche Einstellungen zu Sinn und Zweck von Strafe, dem Verhältnis von Opferschutz und Beschuldigtenrechten sowie der Effektivität völkerrechtlicher Verhaltenssteuerung verstellen allzu leicht den Blick auf das zu begutachtende Objekt; die Erwartungshaltung des Rückblickenden prägt das gezeichnete Bild: Während ein optimistisch gesinnter Betrachter die Anzahl der eingeleiteten Verfahren, die gegen amtierende (al-Bashir) und ehemalige (Gbagbo) Staatschefs erlassenen Haftbefehle und die bereits gefällten Urteile (Lubanga, Chui) betonen und den IStGH als Symbol im Kampf gegen die Straflosigkeit preisen würde, dürfte ein pessimistischer Beobachter die ressourcenintensiven und wenig erfolgversprechenden Verfahren (Kony), die starke Fokussierung auf afrikanische Bürgerkriege fernab des Interesses der ständigen Sicherheitsratsmitglieder (Zentralafrikanische Republik, Demokratische Republik Kongo), Ermittlungen als Legitimationsquelle für militärische Intervention (Libyen), die selektive Auswahl der Täter (Kenia, Uganda) und Taten (Lubanga) sowie rechtsstaatlich zumindest fragwürdiges Verhalten der anklagenden Behörde (Lubanga) unter dem ersten Chefankläger Luis Moreno-Ocampo hervorheben. Erschwert wird der Rückblick durch den zu begutachtenden Wirkungszeitraum. So vermochte der IStGH in der vergangenen Dekade zwar zwei Verfahren durch erstinstanzliche Urteile abzuschließen. Eine gefestigte Rechtsprechung zu umstrittenen Rechtsfragen (z.B. zur Definition der Verbrechen gegen die Menschlichkeit) ist

* Dr. Mayeul Hiéramente, Alumni der International Max Planck Research School on Retaliation, Mediation and Punishment (IMPRS-REMEP). für den juristischen Beobachter indes nicht oder nur schwer auszumachen. Ebenso lässt sich kaum absehen, welche Effekte Verfahren und Urteile auf die betroffene Bevölkerung im Tatortstaat haben werden. Da ein abschließendes Urteil über das Wirken des IStGH in seinem ersten Jahrzehnt mithin verfrüht erscheint, soll dieser Rückblick zum Anlass genommen werden in die Zukunft zu blicken.

Anhand einer rechtspolitisch höchst interessanten Entscheidung der Anklagebehörde des IStGH im Darfur-Verfahren sollen Leitlinien der Entwicklung des Völkerstrafrechts sowie dessen Reformbedürftigkeit aufgezeigt werden. Die Rede ist vom Antrag der Anklage vom 14.7.2008 auf Erlass eines Haftbefehls gegen den amtierenden sudanesischen Präsidenten Omar al-Bashir. Dieser habe sich, so die Anklage, wegen Kriegsverbrechen, Verbrechen gegen die Menschlichkeit und, dies sei hier besonders betont, Völkermord strafbar gemacht. Die zuständige Vorverfahrenskammer I folgte dem Antrag, lehnte aber den Erlass eines Haftbefehls wegen des Verdachts des Völkermordes mangels ausreichender Beweise ab. Nachdem die Berufungskammer festgestellt hatte, dass die Vorverfahrenskammer von einem falschen Beweisstandard ausgegangen war, beantragte die Anklage die Erweiterung des Haftbefehls auf den Verdacht des Völkermordes; am 12.7.2010 erließ die Kammer den erweiterten Haftbefehl.

Auffallend und angesichts der fehlenden Notwendigkeit einer Haftbefehlserweiterung verwunderlich ist, dass sowohl die Anklage als auch zahlreiche Kommentatoren dieser Erweiterung einen derart hohen Stellenwert beizumessen scheinen. ${ }^{1}$ Die

1 Zu Kooperationspflichten aufgrund der Völkermordkonvention siehe Gillett, Matthew, The Call of Justice: Obligations Under the Genocide Convention to Cooperate with the International Criminal Court, in: Criminal Law Forum 1/2012, S. 63-96; Sluiter, Göran, Using the Genocide Convention to Strengthen Cooperation with the ICC in the Al Bashir Case, in: Journal of International Criminal Justice 2/2010, S. 365-382. 
augenscheinliche Betonung des Völkermordsvorwurfs ${ }^{2}$ wirft weitgehende Fragen auf, denen im Folgenden en détail nachgegangen werden soll.

Insbesondere soll diskutiert werden, ob die von einer Vielzahl von Autoren teils intuitiv, teils instrumentalisierend proklamierte Sonderstellung des Völkermords - wahlweise als „crime of crimes", „apex of the pyramid of crimes“, „the ultimate crime" bezeichnet $^{3}$ - auch im 21. Jahrhundert noch gerechtfertigt ist und ob der Fixierung auf Völkermord und die Völkermordkonvention von 1948 entgegengetreten werden sollte. Akhavan hat dazu formuliert: „But, could it be that the prevailing wisdom that genocide is the ultimate crime is wrong? Or that this perception of the crime is a gross oversimplification of a far more complex issue? And could it be that emphasizing the distinctiveness of genocide may actually undermine the very progress and justice that international law intends to achieve?"4 Erhellend und sicherlich ebenfalls zutreffend ist indes der diesem Fragenkatalog folgende Satz: „For some, these questions may border on the international law equivalent of blasphemy. "5 Diesem Überlegungen folgend sollen in den folgenden Abschnitten einige ketzerische Thesen zum Nachdenken über die Frage anregen, ob die Völkermorddefinition für die Tätigkeit des IStGH und generell im 21. Jahrhundert sich als Bereicherung oder als Ballast darstellt.

\section{Die rechtliche Regelung des Völkermords und dessen Schwächen}

Wissenschaftliche Beiträge zum Thema Völkermord beginnen - über die Disziplingrenzen hinweg - auffällig häufig mit der Bekundung eines die Autoren plagenden Unbehagens. Strafrechtler monieren die unbestimmten Konturen des Straftatbestands, Völkerrechtler die mangelnde Handhabbarkeit der Völkermorddefinition für auf Prävention abzielende Rechtsargumentation und Politikwissenschaftler, Historiker und Praktiker die teils willkürliche Auswahl geschützter Gruppen sowie die daraus resultierende Divergenz zwischen Völkermord als Rechtsnorm und dessen sozialer Bedeutung.

\subsection{Völkermord als missglückter Straftatbestand?}

Die Völkermordkonvention von 1948 definiert das strafrechtliche Unrecht des Völkermords in Art. II zunächst wie folgt als „eine der folgenden Handlungen, die in der Absicht begangen wird, eine nationale, ethnische, rassische oder religiöse Gruppe als solche ganz oder teilweise zu zerstören: (a) Tötung von Mitgliedern der Gruppe; (b) Verursachung von schwerem körperlichem oder seelischem Schaden an Mitgliedern der Gruppe; (c) vorsätzliche Auferlegung von Lebensbedingungen für die Gruppe, die

2 Zur vergleichbaren Diskussion hinsichtlich Darfur, Akhavan, Payam, Reducing Genocide to Law, 2012, S. 4, 135f.

3 Überblick bei Akhavan, Fn. 2; S. 10f. Siehe auch Smith, Adam, Genocide and the Europeans, 2010, S. 18 mit dem Fokus auf Prävention.

4 Akhavan, Fn. 2, S. 11.

5 Ebd. Siehe auch Tallgren, Immi, The sensibility and sense of international criminal law, in: European Journal of International Law 3/2002, S. 593: "International Criminal Law carries a kind of religious exercise of hope that is stronger than the desire to face everyday life." geeignet sind, ihre körperliche Zerstörung ganz oder teilweise herbeizuführen; (d) Verhängung von Maßnahmen, die auf die Geburtenverhinderung innerhalb der Gruppe gerichtet sind; (e) gewaltsame Überführung von Kindern der Gruppe in eine andere Gruppe."

Trotz zahlreicher Kritik in der Entstehungsphase der Konvention sowie Schwierigkeiten bei der Interpretation und praktischen Anwendung ist die Definition wortwörtlich in Art. 4 des ICTY-Statuts, Art. 2 des ICTR-Statuts und Art. 6 des IStGH-Statuts aufgenommen worden - ein Vorgehen, das man wahlweise als Kontinuität zur Wahrung der Rechtssicherheit preisen oder als mangelnde Reformierbarkeit werten kann. Sicher ist, dass die Mängel des Völkermordtatbestands durch das Festhalten an der Definition nicht behoben wurden. Exemplarisch dafür mag stehen, dass weder die besondere Dimension der begangenen Verbrechen noch das planhafte Vorgehen der Täter in der gewählten Formulierung zum Ausdruck gebracht werden. ${ }^{6}$ So fordert der Wortlaut ausschließlich die Zerstörungsabsicht des handelnden Täters (special intent) und lässt damit jedenfalls theoretisch die Tat eines Einzeltäters (lone wolf) ausreichen. ${ }^{7}$

Die gesteigerte Bedeutung des subjektiven Tatbestands hat praktische Auswirkungen auf die Beweisfrage. Da selbst in Fällen von Prozessabsprachen (plea bargaining) das Eingeständnis einer Völkermordabsicht eine Rarität ist und vermutlich auch bleiben wird, ist der Nachweis unter Rückgriff auf objektive Indizien nur schwer zu erbringen. Die Anklagepraxis des Internationalen Jugoslawientribunals (ICTY) verdeutlicht die Schwierigkeit und den Preis eines derartigen Unterfangens und zeigt ebenfalls, dass die hohen Erwartungen, die mit einer Völkermordanklage geweckt werden, allzu oft unerfüllt bleiben. Die Schwierigkeit einer derartigen Beweisführung liegt unter anderem in der Tatsache begründet, dass Täter weder de facto noch de jure Rassisten sein müssen. Völkermord ist nicht ipso facto ein Hassverbrechen; er ist vielmehr Mittel zur Verwirklichung diverser Ziele; politische oder militärische Dominanz, Habgier ${ }^{8}$ oder Rache können ebenso Motive der Tat sein wie Rassenhass oder religiöser Fanatismus. Da der special intent vom handlungsleitenden Motiv abzugrenzen ist, ${ }^{9}$ muss ein Rückschluss aus objektiven Kriterien auf die Intention des Täters zwangsläufig schwerfallen. Dies gilt nicht nur für Strafverfahren gegen opportunistische Mitläufer und

6 Zu divergierender Definition im französischen Recht siehe Fournet, Caroline, Reflections on the Seperation of Powers, in: Henham/Behrens(Hrsg.) The Criminal Law of Genocide, 2007, S. 212. Zum Thema auch Cassese, Antonio, The Policy Element in Genocide, in: Safferling/Conze (Hrsg.), The Genocide Convention Sixty Years after its Adoption, 2010, S. 133.

7 Ein guter Überblick über die Debatte findet sich bei Behrens, Paul, A Moment of Kindness?, in: Henham/Behrens(Hrsg.), The Criminal Law of Genocide, 2007, S. 126ff; Cassese, Fn. 6, S. 133ff; Kirsch, Stefan, The Two Notions of Genocide, in: Safferling/Conze (Hrsg.), The Genocide Convention Sixty Years after its Adoption, 2010, S. 145ff.; Schabas, Williams, Has Genocide been Committed in Darfur?, in: Henham/Behrens(Hrsg.), The Criminal Law of Genocide, 2007, S. 42-46. Zur teilweisen, aufgrund der geringen Verfolgungsdichte des IStGH vermutlich praktisch irrelevanten, Einschränkung durch objektive Kriterien in den Elements of Crimes, welche das IStGH-Statut ergänzen, siehe ebd., S. 45. Zur Thematik siehe auch Akhavan, Fn. 2, S.47ff. Slade, Tuiloma, The Prohibition of Genocide under the Legal Instruments of the International Criminal Court, in: Henham/Behrens(Hrsg.), The Criminal Law of Genocide, 2007, S. 157ff.; Kirsch, S. 148.

8 Zum "genocide business" siehe Semelin, Jacques, Towards a Vocabulary of Massacre and Genocide, in: Jones (Hrsg.), Genocide in theory and law, 2008, S. 348.

9 Dazu sehr präzise Behrens, Paul, Genocide and the Question of Motives, in: Journal of International Criminal Justice, 3/2012, S. 501-523 mwN, mit insbesondere lesenswerten Ausführungen zum Verhältnis zu militärischen Zielen, S. $518 \mathrm{ff}$ 
Profiteure. Bestehende Abgrenzungsprobleme zu ethnischen Säuberungen zeigen, ${ }^{10}$ dass der Nachweis der Zerstörungsabsicht selbst bei offenen Befürwortern einer zwangsweisen Durchsetzung „ethnischer Homogenität“ nur mit gesteigertem Aufwand zu erbringen ist. ${ }^{11}$

Weitere Schwierigkeiten treten bei der Bestimmung der Opfergruppen auf. Eindrücklich beschreibt etwa Akhavan die Versuche der Richter am Ruandatribunal (ICTR) im AkayesuVerfahren eine objektive Einordung der Tutsi als „ethnische“ Gruppen zu erreichen. ${ }^{12}$ Der soziale Ursprung der Kategorisierung als Hutu und Tutsi - vergleichbar willkürlich die Zuschreibung „Jude“ im Dritten Reich - in Verbindung mit dem numerus clausus der geschützten Gruppen sowie dem Grundsatz des nulla poena sine lege stricta (keine Strafe ohne Gesetz) erschweren eine objektive Kategorisierung. Das dadurch notwendige teilweise Abstellen auf die Täterintention führt dazu, dass dem Rechtsbrecher die Deutungshoheit weitgehend übertragen wird. ${ }^{13}$ Hinzu kommt, dass durch das Urteil die Gruppeneinteilung quasi zementiert wird. ${ }^{14}$

Trotz der besonderen Kürze und scheinbaren Einfachheit des Völkermordtatbestands ist dieser in der derzeitigen Form als Strafnorm nur bedingt geeignet. Diese Schwächen der Definition setzen sich zudem fort, wenn man den Völkermordtatbestand in seiner Funktion als Präventivnorm betrachtet.

\subsection{Völkermord als missglückte Präventivnorm?}

Spätestens mit den Geschehnissen in Ruanda 1994 und Srebrenica 1995 ist das Thema der Völkermordprävention in das Zentrum des Weltgewissens der Mediengesellschaften katapultiert worden. Die Verhinderung von derartigen Grausamkeiten, mittlerweile durch Spielfilme und Dokumentationen auch der Gesamtbevölkerung bekannt, gilt als zumindest moralische, wenn auch in der Praxis weitgehend ignorierte Pflicht.

Die rechtliche Dimension der in Art. I der Völkermordkonvention nur en passant erwähnten Verhütungspflicht war bis zum Urteil des Internationalen Gerichtshofs (IGH) vom 26.2.2007 (Bosnien und Herzegowina v. Serbien) weitgehend konturen$\operatorname{los}^{15}$ und hat durch die nur teilweise überzeugenden Ausführungen der IGH-Richter keine praxistaugliche Strukturierung erfahren. Dies mag zunächst daran liegen, dass die Prävention bzw. Verhütung von Völkermord zwar im Titel der Konvention und in Art. I Erwähnung findet, indes weder Adressatenkreis

10 Behrens, Fn. 9, S. 516ff; van der Herik, Larissa, The Schism between the Legal and the Social Concept of Genocide in Light of the Responsibility to Protect, in: Henham/Behrens(Hrsg.), The Criminal Law of Genocide, 2007 S. 83f.; Schabas, Fn. 7, S. 40f.

11 Zum Problem der "moments of kindness“ im Rahmen der Beweiswürdigung, Behrens, Fn. 7, S. 128ff. Zum Problem der teilweisen Zerstörungsabsicht siehe Akhavan, Fn. 2, S. 48ff. Zur Problematik des Beweises im Rahmen der Staatenverantwortlichkeit siehe Seibert-Fohr, Anja, The ICJ Jugdement in he Bosnian Genocide case and Beyond, in: Safferling/Conze (Hrsg.), The Genocide Convention Sixty Years after ist Adoption, 2010, S. 252.

12 Akhavan, Fn. 2, S. 149ff. Siehe auch van der Herik, Fn. 10, S. 80 mwN

13 Dazu van der Herik, Fn. 10, S. 89ff.; Vest, Hans, Genozid durch organisatorische Machtapparate, 2002, S. 50,131ff.

$14 \mathrm{Zu}$ den Bemühungen in Ruanda, die Denkstrukturen zu ändern siehe Greenberg, Douglas, Citizenshil, National Identity and Genocide, in: Provost/Akhvan (Hrsg.) Confronting Genocide, 2011, S. 94

15 Dazu etwa Mégret, Frédéric, Not ,Lambs to the Slaughter', in: Provost/Akhvan (Hrsg.) Confronting Genocide, 2011, S. $195 \mathrm{ff}$. noch Umfang der Präventionspflicht explizit benannt werden. So war jedenfalls bis zu besagtem Urteil umstritten, ob auch Drittstaaten eine Rechtspflicht zur Prävention trifft. ${ }^{16}$ Obwohl die IGH-Richter - sicherlich auch aufgrund des geografischen, ideologischen und militärischen Näheverhältnisses zwischen Serbien und den in Bosnien agierenden bosnischen Serben eine Präventionspflicht für Drittstaaten aus dem Wortlaut und Sinn und Zweck der Konvention dem Grunde nach hergeleitet haben, gelang eine Präzisierung des genauen Umfangs nicht bzw. wurde vermieden. ${ }^{17}$

Im hiesigen Kontext sind vor allem drei Feststellungen von Interesse. Erstens, stellten die Richter fest, „[...] that a State can be held responsible for breaching the obligation to prevent genocide only if genocide was actually committed"18. Dies bedeutet, dass selbst bei klaren Anzeichen für drohende Völkermordtaten Untätigkeit nicht als Verstoß gegen Art. I gewertet werden kann, wenn sich die Taten im Nachhinein nicht als Völkermord, sondern „nur" als Verbrechen gegen die Menschlichkeit herausstellen ein deutlicher Anreiz, einer nachträglichen Feststellung des Vorliegens eines Völkermords entgegenzuwirken. Zweitens, betont der IGH, dass das erforderliche Maß des Tätigwerdens von der völkerrechtlichen Zulässigkeit der jeweiligen Maßnahme abhängt. Welche rechtlichen Hürden - die Problematik der sogenannten humanitären Intervention dürfte den Richtern vorgeschwebt haben - indes eine Präventionspflicht ausschließen, wird im Urteil bedauerlicherweise nicht mitgeteilt. ${ }^{19} \mathrm{Ju}$ ristisch zwingend, praktisch jedoch besonders problematisch ist der dritte Punkt: So muss ein Staat Kenntnis der Existenz eines großen Risikos, dass Völkermord begangen werden wird, haben. ${ }^{20}$ Wie aus den obigen Ausführungen deutlich geworden sein sollte, ist die Identifizierung eines special intents - auch im Rahmen einer, nicht die für eine strafrechtliche Verurteilung erforderliche Überzeugung voraussetzende Risikoprognose ein diffiziles Unterfangen. Einblicke in das Innenleben des die Gewalt orchestrierenden Machtapparats sind von außen oft schwer zu erlangen. Das Ausmaß der Gewalt, so es für Drittstaaten feststellbar ist, erlaubt in den meisten unübersichtlichen Konfliktlagen nur selten Rückschlüsse auf die Täterintention. Dies gilt insbesondere im Anbahnungsstadium - ein fundamentaler Unterschied zu den objektiv definierten Verbrechen gegen die Menschlichkeit und Kriegsverbrechen.

\subsection{Norm und Wahrnehmung}

Besonders delikat sind indes nicht die normativen Schwächen, sondern die fragwürdige Sonderbehandlung, die der Völkermordtatbestand im Gefüge des Völkerstrafrechts und des Präventivvölkerrechts erfährt. Mit den Worten Kupers: „the entire field of genocide studies is [...] charged with emotion, selective perception and controversy." 21

16 Zum Völkergewohnheitsrecht siehe van der Herik, Fn. 10, S. 92.

17 Zur Präventionspflicht siehe Hiéramente, Mayeul, Internationale Haftbefehle in noch andauernden Konflikten, 2013, S. 109ff, zur Kritik am Umfang ausführlich S. 111ff. Kritik auch bei Seibert-Fohr, Fn.11, S. $254 \mathrm{ff}$.

18 IGH (Bosnien und Herzegowina v. Serbien), Urteil v. 26.2.2007, para. 430

19 Zur Problematik des Legalitätskriteriums ausführlicher Hiéramente, Fn. 17, S. 113f.

20 IGH, Fn. 18, para. 431

21 Kuper, Leo, The Genocidal State, in: Jones (Hrsg.), Genocide in theory and law, 2008, S. 95. 


\subsubsection{Die Wahrnehmung von Völkermordverfahren}

Trotz weitgehender Unterschiede und teils offensichtlicher Willkürlichkeit in der Herleitung wird Völkermord in Literatur und Rechtsprechung nahezu einhellig als „crime of crimes“ und als schwerstes der im Rom-Statut niedergelegten Verbrechen angesehen. ${ }^{22} \mathrm{Ob}$ diese Sonderstellung gerechtfertigt ist, soll an dieser Stelle nicht vertieft werden. ${ }^{23}$ Stattdessen sollen im Folgenden die inhärenten Gefahren einer derartigen Hierarchisierung anhand einiger Beispiele und grundsätzlicher Erwägungen dargestellt werden. Beweise freilich werden in den folgenden Zeilen nicht zu erwarten sein und so bleibt es den geneigten Leserinnen und Lesern überlassen, die vorgebrachten Argumente zu hinterfragen und zu einer eigenen Einschätzung zu kommen. Weitgehende Einigkeit dürfte jedoch hinsichtlich der Feststellung bestehen, dass Völkermord sowohl in der juristischen Praxis als auch in der medialen und akademischen Bewertung als etwas Anderes, etwas Besonderes und schwer Greifbares angesehen wird.

Ein eindrückliches Beispiel aus der Praxis der Ad-hoc-Tribunale liefert etwa Akhavan mit der Beschreibung der konträren Bemühungen von Richter und Anklage im Fall Jelisic: ${ }^{24} \mathrm{Im}$ besagten Verfahren hatte sich der niederrangige Angeklagte zu einem Geständnis bereit erklärt, das indes den Anklagevorwurf des Völkermords aussparte. Obwohl die Anklage aufgrund dessen eine Einigung ablehnte, sorgten die Richter mit fragwürdiger Begründung für eine rasche Verfahrensbeendigung. Dieses Vorgehen interpretiert Akhavan treffend wie folgt: „Even within the confines of legal proceedings, the legal definition of genocide has thus been supplanted by some larger moral conception of the crime as one requiring a special sort of atrocity, beyond the capacity of mere underlings. " 25

Doch auch das Vorgehen der Anklage verdient Beachtung. Trotz der begrenzten Ressourcen sowie des grundsätzlich durchaus praktizierten charge bargainings ${ }^{26}$ am ICTY scheint die Anklage in diesem und in weiteren Fällen auf die symbolische Bedeutung einer Völkermordanklage nicht verzichten zu wollen. ${ }^{27}$ Ein weiteres interessantes Beispiel aus der Praxis liefert eine Berufungsentscheidung des ICTR in der festgestellt wurde: "The fact of the Rwandan genocide is a part of world history, a fact as certain as any other, a classic instance of a fact of common knowledge." 28 Wie Kirsch treffend anmerkt, ist eine derartige Feststellung im Rahmen eines Strafverfahrens problematisch, ${ }^{29}$ handelt es sich beim Völkermord gemäß der Definition gerade nicht um ein historisches Ereignis, sondern um strafrechtliches Unrecht, sodass der volle

22 Aggression sei aufgrund der bisher mangelnden praktischen Relevanz hier ausgeklammert.

23 Dazu ausführlich Akhavan, Fn . 2, S. 31ff

24 Akhavan, Fn. 2 S. 153ff.

25 Akhavan, Fn. 2, S. 168. Siehe auch van der Herik, Fn. 10, S. 91.

26 Prozessabsprachen, bei denen nicht nur die Strafhöhe, sondern auch die zur Verurteilung führenden Anklagepunkte aushandelbar sind.

27 Vgl auch Dana, Shahram, Genocide, Reconciliation and Sentencing in the Jurisprudence of the ICTY, in: Henham/Behrens(Hrsg.), The Criminal Law of Genocide, 2007, S. 265. Zur Rezeption in den Medien siehe etwa Simons, "Genocide Charge Reinstated Against Wartime Leader of Bosnian Serbs", in: New York Times v. 11.7.2013. Akhavan, Fn. 2, hat zudem herausgearbeitet, dass bei identischer Tatsachengrundlage die Höhe der Strafe vom Tatbestand abhängig ist, vgl. S. 59

28 Zitiert nach Kirsch, Fn. 7, S. 142. Der Nachweis eines „fact of common knowledge" ist im Verfahren nicht erforderlich.

29 Kirsch, Fn. 7, S. 143
Nachweis der Tatbestandsmerkmale im Einzelfall notwendig ist. Doch nicht nur das Thema Verfahrensfairness sticht ins Auge. Viel erschreckender ist die Tatsache, dass Völkermord wie so häufig als ein singuläres Ereignis dargestellt und wahrgenommen wird - der Plural ist aus dem aktiven Wortschatz verschwunden, verdrängt, vergessen. Die Rede ist wahlweise von "the (Rwandan) genocide", "the Genocide" oder dem Holocaust. Selbst das Rom-Statut verwendet für die Ansammlung verschiedener Straftaten in Art. 6 den Singular, während in Art. 7 und 8 die jeweiligen Straftatkataloge treffend in der Pluralform als "crimes" erfasst werden. Werden Verbrechen als „genocidal acts" oder "acts of genocide“ bezeichnet und der Singular vermieden, rumort es heftig hinter den Kulissen. ${ }^{30}$ Eine differenzierte juristische Betrachtung wird unmöglich, die Symbolik überlagert die Rechtsnorm und die Diskrepanz zwischen sozialer Bedeutung und normativem Gehalt wächst.

Ein derartiges Auseinanderfallen zwischen Norm und Wirklichkeit ist, wenn auch für einige Juristen nur schwer zu akzeptieren, nicht per se kritikwürdig. Im Folgenden soll jedoch argumentiert werden, dass das zu beobachtende Auseinanderdriften nicht nur überhöhte Erwartungen zur Folge hat (dazu bereits oben). Es hat darüber hinaus einen Tunnelblick verursacht, der einer unheilvollen selektiven Wahrnehmung förderlich ist und den Blick für das Wesentliche verstellt. Während die Opfer von Srebrenica oder Ausschwitz im kollektiven Gedächtnis der Weltöffentlichkeit fest verankert sind, geraten die Opfer „einfacher" Verbrechen gegen die Menschlichkeit in Vergessenheit. "[It] ends up creating a fetishistic atmosphere in which the masses of bodies that are not to be qualified for the definition of genocide are dumped in a conceptual black hole where they are forgotten."31 Eine derart selektive Erinnerungskultur wird dem Leid der Opfer nicht gerecht, spielt für diese die Intention des Täters an der Spitze des verbrecherischen Machtapparats jedenfalls keine unmittelbare Rolle. So stellt wiederrum Akhavan zu Recht fest, dass man Völkermordtaten zwar aufgrund der besonderen persönlichen Schuld des Täters schwerer bestrafen kann, aber „it is an entirely different matter to suggest that such definition and ranking should determine the anguish of the victim and the deeper meaning attributed to intense suffering."32 Selbst wenn das Opfer aufgrund seiner Gruppenzugehörigkeit ausgewählt wurde und daraus eine besondere psychische Belastung resultieren sollte, wäre eine Ungleichbehandlung nicht gerechtfertigt. So ist der Gruppendimension aufgrund der zum Teil willkürlichen Auswahl der Opfergruppen im Völkermordtatbestand nur unzureichend Rechnung getragen worden. Sie kommt zudem ebenfalls bei anderen Delikten - etwa beim systematischen Angriff auf die Zivilbevölkerung gem. Art. 7 Rom Statut - zum Tragen. Die Prominenz von Völkermordprozessen im Gesamtsystem des Internationalen Strafrechts negiert das gleichwertige Leiden vieler Opfer.

Wie das obige ICTR-Zitat zeigt, verleitet die besondere Symbolik des Genozids Praktiker und Beobachter zudem in be-

30 Zur Reaktion auf den UN-Report Darfur siehe etwa Schabas, Fn. 7, S. 39ff. $\mathrm{Zu}$ Ruanda siehe etwa Akhavan, Fn. 2. S. 3; Dallaire, Romeo/Manocha, Kishan, The Mayor Powers and the Genocide in Rwanda, in: Henham/ Behrens(Hrsg.), The Criminal Law of Genocide, 2007, S. 66; Smith, Fn. 3, S. 162.

31 Charny, zitiert nach Akhavan, Fn. 2, S. 127

32 Akhavan, Fn. 2, S. 130. Ähnlich auch Holmes, in: Jones (Hrsg.), Genocide in theory and law, 2008, S. 195 
sonderem Maße zur Hybris, Geschichte schreiben oder gar die „Wahrheit" herausfinden zu wollen. Die gerichtliche Etablierung von Fakten - oftmals über das für die Beurteilung der strafrechtlichen Verantwortung notwendige Maß bereits hinausgehend - ist augenscheinlich nicht genug. Damit verlassen die Tribunale indes schnell ihnen bekanntes Terrain und machen sich unnötig angreifbar. Forcieren sie die Völkermordanklage, so entsteht schnell der Eindruck der Siegerjustiz - die absolut einseitige Strafverfolgung des ICTR ist das beste Beispiel. ${ }^{33}$ Lässt die Anklage den Anklagevorwurf fallen, wird ihr vorgeworfen Versöhnung zu verhindern und Revisionismus Vorschub zu leisten. ${ }^{34}$ Jonassohn hat ein Charakteristikum des Völkermords, das augenscheinlich in vielen Köpfen verankert ist, zutreffend herausgearbeitet und bezeichnet diesen als „a form of one-sided killing“ . ${ }^{35}$ In der Wahrnehmung lässt Völkermord nur Raum für Opfer oder Täter bzw. Opferund Tätergruppen. Dies läuft dem Grundgedanken der Individualisierung von Schuld oft diametral entgegen und droht Freund- und Feindbilder zu zementieren anstatt ihnen entgegenzuwirken. Die Täter als Gruppe zu sehen, welcher die Verantwortung für „den“ Völkermord zuzuschreiben ist, droht das komplexe Zusammenwirken zwischen rassistischen oder religiösen Fanatikern, gehorsamen Verwaltern, machthungrigen Opportunisten und Mitläufern zu verdecken. ${ }^{36}$ Aus einer Ansammlung verschiedener Motive und Handlungen wird ein künstlich erschaffenes singuläres Ereignis, das außerhalb des Gerichtssaals kaum Raum für eine differenzierte und individualisierte Wahrnehmung lässt. ${ }^{37}$ Die kollektive Interpretation lässt zudem das Leiden des Individuums in den Hintergrund treten und degradiert es zum schutzlosen Opfer. ${ }^{38}$ Es lebt mithin die Kritik fort, die Hannah Arendt so treffend gegenüber dem Eichmannprozess in Jerusalem geäußert hat. ${ }^{39}$ Statt einem Verbrechen gegen Menschen, Menschlichkeit oder Menschheit, sehen wir nur das Verbrechen gegen Juden oder Tutsis.

\subsubsection{Reden statt Handeln}

Auch im Bereich der Völkermordprävention scheint der Fokus auf das G-Wort das Wesentliche zu verdrängen. Insbesondere aus der Opferperspektive muss das sich ständig wiederholende Feilschen um Worthülsen angesichts der vor Ort durchlebten Grausamkeiten absurd anmuten. Es ist daher zu begrüßen, dass jedenfalls seitens der UN im Bereich der Prävention von schwersten Verbrechen z.B. durch die Verabschiedung der

33 Zur Thematik Smith, Fn. 3, S. 167. Siehe auch die einseitige Wahrnehmung in Filmen wie „Hotel Ruanda“ oder "Sometimes in April“.

34 Siehe Dana, Fn. 27, S. 267.

35 Jonassohn, Kurt, What is Genocide?, in: Jones (Hrsg.), Genocide in theory and law, 2008, S. 117. Ähnlich Levene, Mark, Why ist he Thwentieth Century the Century of Genocide, in: Jones (Hrsg.), Genocide in theory and law, 2008, S. 294; Bauer, Yahuda, Some Problems of Genocide Prevention, in: Provost/Akhvan (Hrsg.) Confronting Genocide, 2011, S. 108. Zur problematischen Einordnung der Opfer als schwach und hilflos siehe Mégret, Fn. 15, S. $203 f$.

36 Ausführlich Vest, Fn. 13, S. 31ff.

37 Siehe auch Byamukama, Shivon/Huntley, John, Criminal Justice in the Aftermath of 1994 Rwanda Genocide, in: Henham/Behrens (Hrsg.), The Criminal Law of Genocide, 2007, S. 231: „It is simplistic to conceive the genocide as one specific event. It is a period within a series of events in a troubled history." Gute Beschreibung der Problematik bei Straus, Scott, The Order of Genocide, in: Jones (Hrsg.), Perpetrators, Victims, Bystanders, Rescuers, 2008, S. 246ff.

38 Dazu Mégret, Fn. 15, S. 228 f.

39 Siehe dazu auch Akhavan, Fn. 2, S. $118 \mathrm{ff}$ m.w.N..
Responsibility to Protect und der Erweiterung des Mandats des Special Advisors on the Prevention of Genocide ein inklusiverer Ansatz vertreten wird. Doch selbst wiederholte Hinweise, dass „international offences as crimes against humanity and large scale war crimes may be no less serious and heinous than genocide" 40 die Notwendigkeit eines derartigen Hinweises verdeutlicht dies eindrücklich - haben nur bedingt zu einem Umdenken geführt. So stellt Schabas die Reaktion auf den Bericht der Darfur-Kommission treffend dar, wenn er schreibt: „Many human rights activists were disappointed by the conclusion that genocide had not been committed, and some went so far as to see the Commission report as some kind of whitewash or even betrayal." ${ }^{41}$ Ähnliches merkt Kaufmann in einer Analyse zur Debatte innerhalb der US-Administration hinsichtlich Darfur an. Die Bezeichnung als Genozid bedeute - auch aufgrund der Völkermordkonvention - eine gesteigerte Erwartung hinsichtlich eines zukünftigen Engagements. ${ }^{42}$ Ebenso bedauern Dallaire und Manocha noch in einem 2007 erschienen Aufsatz, dass sich die USA, Großbritannien und Frankreich der Bezeichnung der Massaker in Ruanda als Völkermord so lange entgegengestellt haben. ${ }^{43}$

Während man das Bestreben der Praktiker nachvollziehen kann, aufgrund der besonderen Symbolik des Terminus Genozid, derartige Wortklauberei zu betreiben, muss man sich der Nachteile bewusst sein. Die Intention der Täter und deren Nachweisbarkeit bestimmen mit, ob und wie im Interesse der Opfer reagiert wird. ${ }^{44}$ Akhavan hat daher hinsichtlich der Reaktion auf die Geschehnisse in Ruanda angemerkt: „It is striking that both proponents and opponents of intervention continually focused the debate on whether the events in Rwanda amounted to genocide'. This emphasis gave such a privileged place to hierarchical abstractions and formal reasoning that the horrors unfolding before the eyes of the world were overshadowed. " 45 Der UN-Sonderberichterstatter für die Verhinderung des Völkermords Méndez hat die Problematik auf den Punkt gebracht: „Too often the debate over whether genocide is occurring has become more important than taking action to reverse the situation and prevent further violations. "46

\section{Völkermordregeln abschaffen: Ein Gedankenexperiment}

Der Völkermordtatbestand sowie dessen Instrumentalisierung durch Politik und Medien wurden von jeher kritisiert. Bereits

40 Report of the International Commission of Inquiry on violations of international humanitarian law and human rights law in Darfur, UN Doc. S/2006/60, para. 533.

41 Schabas, Fn. 7, S. 39; siehe auch van der Herik, Fn. 10, S. 88.

42 Kaufmann, Zachary, Sudan, the United States and the International Criminal Court, in: Henham/Behrens (Hrsg.), The Criminal Law of Genocide, 2007, S. 58. Siehe Smith, Fn. 3, S. 104, 154. Hamilton, Rebecca, Creating the Outcry, in: Provost/Akhvan (Hrsg.) Confronting Genocide, 2011, S. 272 betont indes das Risiko, dass die Schwere der Vorwürfe bei Bürgern auch zu resignierendem Stillstand führen kann.

43 Dallaire/Manocha, Fn. 30, S. 71. Guter Überblick auch bei Smith, Fn. 3, S. $65 \mathrm{ff}$.

44 Sofern nicht bereits realpolitische Erwägungen ein Tätigwerden verhindern Zur Gefahr, dass sich Regierungen hinter der engen Definition verstecken, siehe Smith, Fn. 3, S. 8.

45 Akhavan, Fn. 2, S. 139; zu Darfur, S. 3. Ähnliche Einschätzung zu Darfur bei Smith, Fn. 3, S. 208ff.

46 Méndez, Juan, The United Nations and the Prevention of Genocide, in: Henham/Behrens (Hrsg.), The Criminal Law of Genocide, 2007, S. 288. Ähnlich Mégret, Fn. 15, S. 200f. Smith, Fn. 3, S. 8 fragt gar, ob nicht die Benennung das Handeln ersetzt. 
im Rahmen der Verhandlungen zur Völkermordkonvention vertraten französische Unterhändler die Auffassung es handele sich beim Begriff Völkermord um ein „useless and even dangerous neologism". ${ }^{47}$ Auch heute - 65 Jahre später - verzichtet kaum ein wissenschaftlicher Artikel auf kritische Einführungen hinsichtlich der Definition und deren Interpretation durch Juristen und juristische Laien sowie die Feststellung, dass "the Genocide Convention has been more honored in the breach than in the practice ${ }^{\prime 48}$. Dem wissenschaftlichen Interesse und der Faszination der Beobachter scheint dies keinen Abbruch zu tun. Kommentare, Monografien, Sammelbände und Fachzeitschriften füllen regalweise Bibliotheken. ${ }^{49}$ Umso mehr muss es verwundern, dass anscheinend nur vergleichsweise selten Konsequenzen aus der teils wohlwollenden teils massiven Kritik gezogen wird. Scheffer hat indes vorgeschlagen, im allgemeinen Sprachgebrauch auf weniger polarisierende Begriffe zurückzugreifen und die Verbrechen gem. Art. 6-8 des Rom-Statuts pauschal als „atrocity crimes" zu bezeichnen. ${ }^{50}$ Ähnlich betonen verschiedene Autoren die Notwendigkeit einer deutlicheren Trennung zwischen der Rechtsnorm und deren sozialer Bedeutung. Van der Herik schlägt darüber hinaus vor, Völkermord im strafrechtlichen Sinne von der Präventivnorm gedanklich und sprachlich abzukoppeln. ${ }^{51}$

Vorliegend soll ein radikaleres Gedankenexperiment vorgeschlagen werden: die Abschaffung der Völkermordkonvention und die Streichung des Völkermordtatbestands aus internationalen Statuten.

\subsection{Unersetzbarkeit des Völkermordtatbestands?}

Manch einer wird mit Fug und Recht fragen, ob die Errungenschaften der Völkermordkonvention aufgrund der oben beschriebenen „Petitessen“ und „Unannehmlichkeiten“ geopfert werden sollten; ob nicht eine Lücke klaffen würde und ein zivilisatorischer und juristischer Rückschritt zu befürchten sei. Dem soll im Folgenden in gebotener Kürze auf den Grund gegangen werden.

Nur auf den ersten Blick steht zu befürchten, dass im Falle einer Abschaffung des Völkermordtatbestands die notwendige Bestrafung von Menschheitsverbrechern durch internationale Straftribunale zu scheitern droht. Aufgrund der „Emanzipierung" der Verbrechen gegen die Menschlichkeit - Loslösung vom bewaffneten Konflikt ${ }^{52}$ und der Aufgabe der Staatszentriertheit ${ }^{53}$ - ist deren Anwendungsbereich bedeutend erweitert worden. Auch die Notwendigkeit einer „Politik eines Staates oder einer Organisation“ (state or organizational policy) in Art. 7 des Rom-Statuts, die Straftaten durch sog. lone wolves ausschließt, dürfte aufgrund der stärkeren Fokussierung auf Führungsfiguren (siehe etwa das Kriterium der Schwere in

47 Zitiert nach Smith, Fn. 3, S. 39.

48 Levene, Fn. 35, S. 288.

49 Überblick bei Akhavan, Fn. 2, S. 9.

50 Dazu Smith, Fn. 3, S. 19.

51 Van der Herik, Fn. 10, S. 94

52 Siehe etwa Vest, Fn. 13, S. 75 ff. Auf S. 55 ordnet Vest zudem Verbrechen gegen die Menschlichkeit als Grundtatbestand ein.

53 Zur Debatte Werle/Burghardt, in: Zeitschrift für internationale Strafrechtsdogmatik, 6/2012, S. 271-281. Zur Entwicklung des Tatbestandsmerkmals siehe auch Akhavan, Fn. 2, S. $37 \mathrm{ff}$.
Art. 17 (1) (d) des Rom-Statuts) in der Praxis nicht zu Strafbarkeitslücken führen. So verwundert es auch nicht, dass sämtliche Völkermordurteile am ICTY ebenfalls Verurteilungen wegen weiterer Verbrechen enthalten. Auch ein kursorischer Blick auf die Rechtsprechung des ICTR zeigt, dass eine Verurteilung ausschließlich wegen Völkermords eine absolute Rarität ist und sich weitgehend auf die Anklagepraxis, ${ }^{54}$ Prozessabsprachen ${ }^{55}$ oder das von den Richtern angenommene Rangverhältnis zwischen Völkermord und Verbrechen gegen die Menschlichkeit ${ }^{56}$ zurückführen lässt. Zwei Verfahren stechen indes heraus und verdienen eine nähere Betrachtung: Bikindi und Muvunyi. Beide wurden ausschließlich wegen „Aufstachelung zur Begehung von Völkermord“ (incitement to commit genocide) verurteilt, eine Begehungsform die es nach derzeitiger Rechtslage (vgl. Art. 25 (3) (e) des Rom-Statuts) bei Verbrechen gegen die Menschlichkeit und Kriegsverbrechen nicht gibt. ${ }^{57}$ Von der Erkenntnis geleitet, dass Hasspropaganda notwendige Bedingung für menschenverachtende Verbrechen ist und „that words are deeds" 58 , haben die Gründungsväter der Völkermordkonvention auch Maßnahmen weit im Vorfeld der Gewalttaten unter Strafe gestellt. Diese Errungenschaft ist bis dato eng mit dem Völkermordtatbestand verbunden gewesen und sollte nicht leichtfertig aufgegeben werden. Daher dürfte es sich empfehlen, die Beteiligungsform auf das allgemeinere Delikt - Verbrechen gegen die Menschlichkeit - auszuweiten. ${ }^{59}$ Abschließend lässt sich demnach feststellen, dass das strafrechtliche Instrumentarium zur strafrechtlichen Ahndung der schwersten Verbrechen auch ohne den Völkermordtatbestand weitgehend ausreichend ist. ${ }^{60}$

Ein wenig schwerer dürfte es fallen, im Bereich der Prävention auf die Völkermordkonvention und deren Instrumentarien $\mathrm{zu}$ verzichten. So ist derzeit einzig in deren Art. I eine extraterritoriale Schutzpflicht verankert, deren Beachtung zudem noch im Rahmen eines IGH-Verfahrens eingefordert werden kann. Tatsache ist jedoch, dass der Umfang der oben diskutierten Schutzpflicht nur in seltensten Fällen ein Tätigwerden des Auslands rechtlich einzufordern vermag. Deshalb und aufgrund der langen Verfahrensdauer vor dem $\mathrm{IGH}^{61}$ ist die praktische Bedeutung der Konvention für den Bereich der Prävention äußerst gering. Die rechtlichen Regelungen - sei es Strafrecht oder das Recht der Staatenverantwortlichkeit - sind

54 So war in den Verfahren Kalimanzira, Ntakirutimana und Ntawukulilyayo das inkriminerte Verhalten nur als Völkermord angeklagt.

55 Verfahren gegen Bagaragaza.

56 Während z.B. in den Verfahren Akayesu und Nsengiyumva ein (!) Verhalten zur Verurteilung wegen mehrerer Straftatbestände geführt hat (siehe dazu etwa Akhavan, Fn. 2, S. 52ff), vertraten die Richter in Kayishema und Ruzindana die Ansicht, dass Völkermord die anderen Straftatbestände verdrängt.

$57 \mathrm{Zu}$ "Aufstachelung" siehe Salomon, Tory, Freedom of Speech v. hate Speech, in: Henham/Behrens(Hrsg.), The Criminal Law of Genocide, 2007, S. 141ff; Slade, Fn. 7, S. 156f.; Cotler, Irwin, Combating State-Sanctioned Incitement to Genocide, in: Provost/Akhvan (Hrsg.) Confronting Genocide, 2011, S. 134ff; Vest, Fn. 13, S. 189ff. Zu Divergenzen bei den Begehungsformen siehe ausführlich Karnavas, Michael, Is the Emerging Jurisprudenceon Complicity in Genocide before the International Ad Hoc Tribunals a Moving Target in Conflict with the Principle of Legality?, in: Henham/ Behrens(Hrsg), The Criminal Law of Genocide, 2007,S. 99ff.

58 Salomon, Fn. 57, S. 142. Kritisch Straus, Fn. 37, S. 251.

59 Dabei ist relevant, dass gerade nicht hate speech als solche unter Strafe gestellt ist; vgl. Salomon, Fn. 57, S. 143.

60 Im Übrigen regeln die Art. 77ff des Rom-Statuts den Strafrahmen einheitlich.

61 Zur Wahrscheinlichkeit von Verfahren siehe etwas Smith, Fn. 3, S. 87f., 93f., 97, 109. 
ausschließlich reaktiv. ${ }^{62}$ Praktisch vielversprechender erscheinen daher die im Kern politisch-moralischen Verpflichtungen der Responsibility to Protect. Das Verfahren Bosnien und Herzegowina gegen Serbien hat gezeigt, dass die Anwendung und gerichtliche Durchsetzung der Konventionspflichten primär symbolischer Natur sind.

Damit ist ein zentraler Aspekt angesprochen. So scheint es vor allem die symbolische Bedeutung des Terminus zu sein, die intuitiv dazu verleitet an diesem festzuhalten. Erfordert nicht die ständige Reizüberflutung einen Begriff, der heraussticht und wachrüttelt, der die Regierungen zum Handeln drängt und vergangenes Leid angemessen würdigt? Ist es erforderlich, dass wir ein konkretes Leid einer abstrakten Kategorie zuordnen anstatt das Leid als solches zu würdigen ${ }^{63}$ Erscheint es sinnvoll, dass ein Gerichtsverfahren gegen einen ruandischen Verbrecher nicht vor norwegischen Gerichten durchgeführt werden konnte, weil das norwegische Strafrecht zu diesem Zeitpunkt die Tat zwar angemessen bestrafen und die besondere Gesinnung des Täters berücksichtigen konnte, der Straftatbestand aber mit Mord und nicht Völkermord überschrieben war? ${ }^{64}$ Diese Fragen mag ein jeder für sich selbst beantworten. Wichtig ist jedoch zu erkennen, dass Völkermord nicht aus sich selbst heraus eine Sonderstellung einnimmt. Politiker, NGOs, Richter, Staatsanwälte und Wissenschaftler wecken Erwartungen, schreiben dem Terminus besondere Eigenschaften zu und haben aus einem Sammelsurium von „namenlosen Verbrechen“ ein Symbol erschaffen. Wenn etwa darauf verwiesen wird, dass Opfergruppen eine Strafverfolgung wegen Völkermord erwarten, so zeigt dies nicht unbedingt die besondere Schwere des Genozids. Es zeigt vielmehr, wie Erwartungshaltungen geschaffen werden und zu einem Wettbewerb um Anerkennung führen können. ${ }^{65}$ Jedes Plädoyer für die Beibehaltung der Völkermordsymbolik ist eine Form der self-fullfilling prophecy, eine zirkuläre Selbstbestätigung. Diesen Kreislauf zu unterbrechen ist daher Ziel des hiesigen Gedankenexperiments.

\subsection{Reform oder Abschaffung?}

Schwächen einer juristischen Definition und das Auseinanderfallen von Rechtsnorm und Wirklichkeit müssen nicht stets die Abschaffung der Norm zur Folge haben. Allerdings haben die letzten sechs Jahrzehnte deutlich gezeigt, dass der Völkermordtatbestand nicht reformierbar ist und die Divergenz zwischen rechtlichem Inhalt und Wahrnehmung fortbesteht. ${ }^{66}$ So ist es kein Zufall, dass die Tatbestände Verbrechen gegen die Menschlichkeit und Kriegsverbrechen kontinuierlich optimiert worden sind, während beim Völkermordtatbestand keine nennenswerten Anpassungen erfolgt sind. Weitgehende Reformen des Tatbestands sind schlichtweg kaum vorstellbar. Eine Erweiterung der geschützten Gruppen erscheint zwar

62 Mégret, Fn. 15, S. 197f mwN.

63 So wohl Fournet, Fn. 6, S. 219.

64 Siehe ICTR (Prosecutor $v$. Michel Bagaragaza), Case No. ICTR-2005-86R11bis, Decision on the Prosecution Motion for Referral to the Kingdom of Norway, Rule 11 bis of the Rules of Procedure and Evidence, 19.5.2006.

65 Dazu Akhavan, Fn. 2, S. 124.

66 Ebenso pessimistisch Akhavan, Fn. 2, S. 147, aber mit weniger radikalen Forderungen, S. 174, 177, 179. grundsätzlich möglich. Selbst solche kosmetische Reformen ${ }^{67}$ vermögen die Problematik der Vorsatzlastigkeit und die daraus resultierenden Beweisschwierigkeiten jedoch nicht zu beheben. Eine, dem etymologischen Ursprung des Terminus Genozid eher entsprechende, objektive Definition ist angesichts der in der Praxis glücklicherweise nur partiell zu konstatierenden Vernichtung bedrohter Gruppen nur schwer vorstellbar. ${ }^{68}$ Ebenso erscheinen die wiederholten Forderungen aus Praxis und Wissenschaft, man solle andere Formen des Systemunrechts nicht ignorieren, angesichts der mantrahaften Hervorhebung des primus inter pares illusorisch. Der Terminus Völkermord/Genozid übt eine Strahlkraft aus, die blendet, trügt und Umrisse verschwimmen lässt. Dieser Glanz soll ihm durch die Abschaffung der rechtlichen Grundlagen genommen werden: Der Begriff selbst kann und soll u.a. für die historische, politikwissenschaftliche und politische Auseinandersetzung erhalten bleiben. Das unheilvolle Wechselspiel zwischen rechtlicher und sozialer Norm sollte hingegen unterbunden werden. ${ }^{69}$

\section{Plädoyer für das Ende einer Dystopie}

Raphaël Lemkin und seine Mitstreiter haben - die hitlersche Vernichtungsmaschinerie im Hinterkopf - mit der Verabschiedung der Völkermordkonvention von 1948 einer Dystopie zu ihrer Verrechtlichung und Verankerung im kollektiven Gedächtnis verholfen. Sie haben damit einen wichtigen Beitrag zur Fortentwicklung des Völker(straf)rechts geleistet und den Grundstein für eine Internationalisierung der Strafverfolgung gelegt. So ist es auch den Bemühungen Lemkins zu verdanken, dass das verursachte menschliche Leid in den Vordergrund gerückt wurde und eine militärisch-strategische Sichtweise insbesondere der Fokus auf die (unerlaubte) Kriegführung ihre prominente Rolle verloren hat. Doch haben sich die Rahmenbedingungen in den letzten Jahrzehnten verändert. Zwischenstaatliche Kriege sind seltener geworden, innerstaatliche Konflikte scheinen komplizierter und die internationale Betroffenheit ist leichter zu mobilisieren. Das Völkerstrafrecht ist aus den Kinderschuhen herausgewachsen, in den letzten zwanzig Jahren mit Siebenmeilenstiefeln vorangeschritten und mit der ständigen Institution des IStGH fest auf der Weltbühne verankert. Steigbügel wie die Völkermordkonvention und die Parallelregelungen in den Statuten sind keine Notwendigkeit mehr; sie sind weitgehend unnötiger Ballast.

67 Siehe z.B. Paul, Angela, Kritische Analyse und Reformvorschlag zu Art. II Genozidkonvention, 2008, S. 211ff.

68 Vgl. Valentino, Benjamin, Bass Killing and Genocide, in: Jones (Hrsg.), Genocide in theory and law, 2008, S. 218. Auch eine quantitative bzw. rein objektiv definierte Teilvernichtung ist kaum vorstellbar.

69 Zur Bedeutung der rechtlichen Grundlage für eine deklarierte Sonderstellung siehe Mégret, Fn. 15, S. 228; Smith, Fn. 3, S. 7. 